MATEC Web of Conferences 51, 02011 (2016)

DOI: $10.1051 /$ matecconf/20165102011

(C) Owned by the authors, published by EDP Sciences, 2016

\title{
EEG Controlled Wheelchair
}

\author{
Sim Kok Swee ${ }^{1, a}$, Kho Desmond Teck Kiang ${ }^{1}$ and Lim Zheng You ${ }^{1}$ \\ ${ }^{1}$ Faculty of Engineering and Technology, Multimedia University, Malaysia
}

\begin{abstract}
This paper describes the development of a brainwave controlled wheelchair. The main objective of this project is to construct a wheelchair which can be directly controlled by the brain without requires any physical feedback as controlling input from the user. The method employed in this project is the Brain-computer Interface (BCI), which enables direct communication between the brain and the electrical wheelchair. The best method for recording the brain's activity is electroencephalogram (EEG). EEG signal is also known as brainwaves signal. The device that used for capturing the EEG signal is the Emotiv EPOC headset. This headset is able to transmit the EEG signal wirelessly via Bluetooth to the PC (personal computer). By using the PC software, the EEG signals are processed and converted into mental command. According to the mental command (e.g. forward, left...) obtained, the output electrical signal is sent out to the electrical wheelchair to perform the desired movement. Thus, in this project, a computer software is developed for translating the EEG signal into mental commands and transmitting out the controlling signal wirelessly to the electrical wheelchair.
\end{abstract}

\section{Introduction}

At the present time, the amount of disabled person is increasing annually in Malaysia. By referring to the statistical data that obtained from the Department of Social Welfare Malaysia, The information of the statistical data shows that the amount of physical disabled person are rising from year 2008 to 2011[1]. According to the statistics data, there are total of 657,152 people with disabilities registered in Malaysia calculated from 2009 until 2013[2] [3] [4] [5] [6].

According to the statistical data in 2013, there are 162,215 persons suffered from physical disability among 494,074 number of disabled person [6]. The percentage of physically disabled persons among all the disabled person is 33\%, which is the second majority of the disabled person [6]. Most of these physically disabled persons are facing the difficulty to move around freely.

Since the physically disabled person is one of the major contribution to total number of disabled persons, this indicates that the amount of disabled persons who lost their mobility are substantial. Wheelchair is the most common device that used to provide mobility for the physically disabled person. However, most of the wheelchair today especially the affordable manual wheelchair requires human power to maneuver. Even for the electrical wheelchair, it still requires user's finger to move the joystick or press the button in order to control the movement of the electrical wheelchair [7]. In this way, some users who completely lost their hands or those who having the difficulty to control their hands such as Poliomyelitis patients are not able to navigate the wheelchair movement. Hence, they are incapable to move around by themselves. With the intention to resolve this issue, another

${ }^{\text {a }}$ Corresponding author : kssim@mmu.edu.my 
constructive way is by using the brain to directly control the movement of the wheelchair. This method will allow most of the people to navigate the wheelchair by themselves. Therefore, this will bring an extremely high impact, especially for the disabled individuals who are not able to communicate physically [8].

The activities of the brain are required to be analyzed for implementing the Brain-computer interface (BCI) for the wheelchair. According to the technology nowadays. There are several methods that can be utilized to obtain the signals of brain activities. These methods are known as Single Photon Emission Tomography, Positron Emission Tomography [9], Functional Magnetic Resonance Imaging and Electroencephalogram (EEG). However, EEG is the most suitable method to be utilized for retrieving the signal of brain activity in order to implement the Brain-computer interface system. This is because the EEG device is portable and non invasive [10].

EEG is a method that captures electrical waveform from the brain. This method involves the EEG device that equipped with electrodes. After appropriately placed these electrodes on the scalp, the voltage fluctuations resulting from ionic current within the neurons of the brain are measured [11]. The electrical motivations resulted from the nerves of the brain are recorded using this method [12]. According to the human thoughts, different electrical waves are produced. Even for a muscle contraction, it will also generate a unique electrical signal [13]. By using the EEG device, these signals are captured and will be transmitted to the computer for further processing.

The patterns of the electrical waves that produced by the brain is distinctive due to different kind of thoughts. Therefore, during the EEG signal processing, the raw EEG signals are processed in a manner to differentiate the mental commands that are thinking by the user. The examples of these commands are forward, backward, left, right and stop [14]. After obtaining the mental command, it is used to produce the electrical control signals for controlling the movement of electrical wheelchair.

The method of translating the brain signals into controlling signals extends to the study of BCI [15]. BCI is a direct communication between the brain and a device that enables signals from the brain to direct some external activity. In this project, the signal from the brain is captured and transmitted to control the external device, which in this case is the electrical wheelchair.

\section{Design and Implementation}

\subsection{Methodology}

An electrical wheelchair is required for this project. The electrical wheelchair structure is built from scratch and the electronic circuits are designed in order to control the electric motor on the wheelchair. The electronic circuits include the microcontroller and motor driver. The connections of the electronic boards are connected as shown in the block diagram in Figure 1.

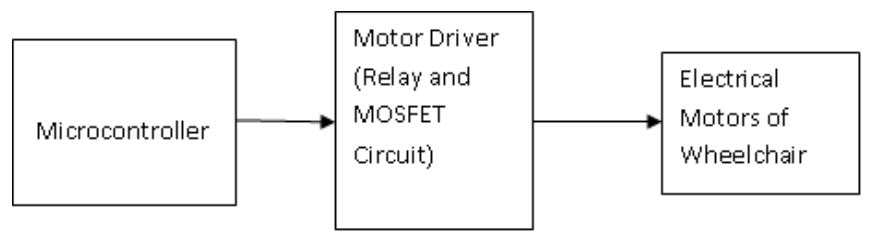

Figure 1. Electrical Board Connection in Electrical Wheelchair System

The whole BCI system consists of several devices. These devices are known as the Emotiv EPOC headset, laptop or PC with Windows operating system and electrical wheelchair with a microcontroller. The interconnection between the devices is illustrated in the Figure 2. 


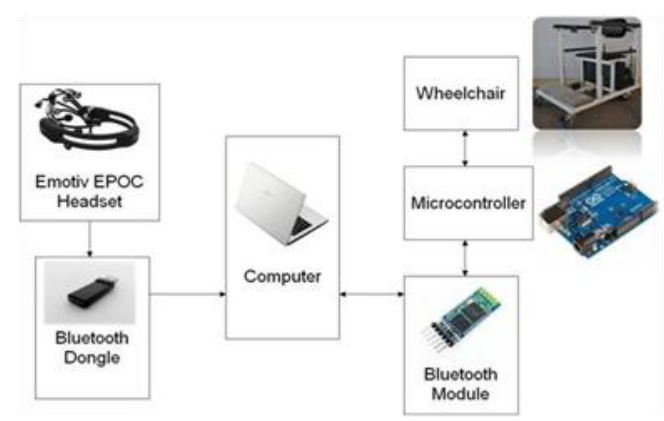

Figure 2. Block Diagram of Brainwave Controlled

\subsection{Selection of EEG Device}

With the intention to realize the Brain-computer interface $(\mathrm{BCI})$, a device is required to capture the information of the brain activity. The method proposed for obtaining the brain activity signal is the Electroencephalogram (EEG). Therefore, it is necessary to choose the most suitable EEG device for this project. There are several EEG devices that can be found in the commercialized market. They are known as OpenEEG, Mattel Mindflex, Neurosky Mindset and Emotiv EPOC.

In order to implementing the BCI for the electrical wheelchair, there are few requirements that have to be fulfilled by the EEG device. First, the device must be portable and convenient. In this case, the OpenEEG is not suitable as the electronic boards involved are space consuming[16]. Beside OpenEEG device, the other devices are portable and wearable. Another requirement for this BCI is the software developer tool. As EEG is a complicated electrical waveform, it will be very time consuming for developing a software that is able to interpret and process these waveforms from fundamental. The provided developer tool is able to lighten the developer's burden. This is because the developer tool contains the basic algorithm to process the raw EEG waveform, such as Fast Fourier Transform. After undergo the fundamental signal processing, the developer is able to acquire the desired data based on the BCI purpose. In this case, there are only Neurosky Mindset and Emotiv EPOC provide developer tool. Therefore, Mattel Mindflex headset is not chosen for this project. In reviewing the Neurosky Mindset and Emotiv EPOC, the major difference between Emotiv EPOC and Mindset is the number of sensors and the location of the sensors. Emotiv EPOC has 14 sensors placed all over the head while the Mindset has only one sensor located on the forehead. With these sensors, Emotiv EPOC is able to detect the strength of signal in every different part of the brain. The Mindset only relies on one sensor to capture the EEG signal, which can only monitor the activity in one part of the brain. Therefore, Emotiv EPOC is able to provide more accurate data compare to the Neurosky Mindset. In addition, Emotiv EPOC is able to monitor the whole brain activity pattern. This feature is very useful for this project to differentiate the brain activity pattern that associates to the mental command which is thinking by the user. Therefore, Emotiv EPOC is the most ideal EEG device for this project.

\subsection{Hardware}

A wheelchair structure with size of 36 inches long and 24 inches wide is designed. The wheelchair is rear wheel differential drive. The structure of the wheelchair is constructed by using the material of steel. 


\section{MATEC Web of Conferences}

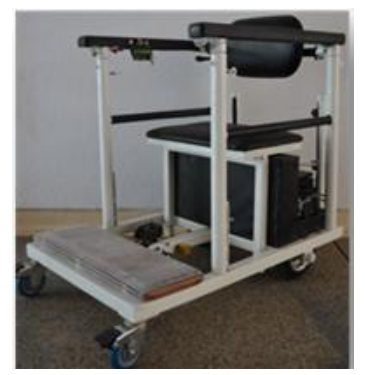

Figure 3. The Structure of Wheelchair.

In order to drive the wheelchair with a person on it, high torque motors are required. Therefore, scooter motors are chosen to drive the rear wheels of the wheelchair. The maximum voltage for this motor is DC $24 \mathrm{~V}$. The power rating for the motor is $250 \mathrm{~W}$. The speed of the motor is $3000 \mathrm{rpm}$ and its rated torque is $0.80 \mathrm{Nm}$. This motor draws maximum current up to $13.4 \mathrm{~A}$.

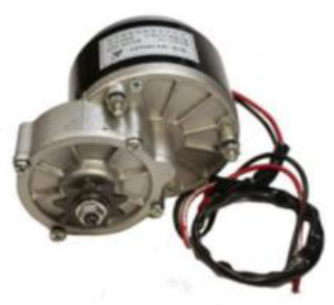

Figure 4. Scooter Motor

As the scooter motor draws high current, a motor driver which is able to sustain high current is required for driving the motors. The scooter motor will draw high current up to $13.4 \mathrm{~A}$, therefore the current rating for motor driver must exceed the maximum current of the DC motor. The chosen motor driver is high current motor driver. This motor driver can support DC $12 \mathrm{~V}$ to $30 \mathrm{~V}$. It can support up to $160 \mathrm{~A}$ peak current and $60 \mathrm{~A}$ continuous current. Thus, this motor driver is very suitable for driving the scooter motor of the wheelchair. Figure 5 shows the high current motor driver.

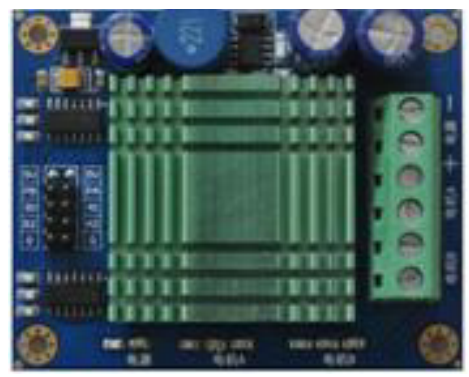

Figure 5. High Current Motor Driver.

Arduino Uno microcontroller board is using ATmega328P microcontroller IC. This microcontroller has 14 digital I/O pins and 6 analog inputs. Within these 14 digital I/O pins, 6 of them can be directly configured as PWM output. It also has $16 \mathrm{MHz}$ quartz crystal on this microcontroller board. This board also consists voltage regulator IC which can support input voltage from 6 to $20 \mathrm{~V}$. The dimension of this microcontroller board is $68.6 \mathrm{~mm} \times 53.4 \mathrm{~mm}$. This microcontroller board is selected because it consists enough number of I/O pins to control the electrical wheelchair. The entire wheelchair has 4 switches as input. The motor driver as described earlier requires 4 output pins and 2 
PWM pins as control signals. In this way, four analog pins are used for input switches, four digital output pins and two PWM pins are used for controlling the motor driver. Besides, this microcontroller is relatively small size, thus it is convenient to be mounted on the wheelchair. In addition, this microcontroller has the feature of plug and play; it only requires a USB type B cable for both programming and serial interface purpose. Figure 6 shows the Arduino Microcontroller Board that used as the main controller of the electrical wheelchair.

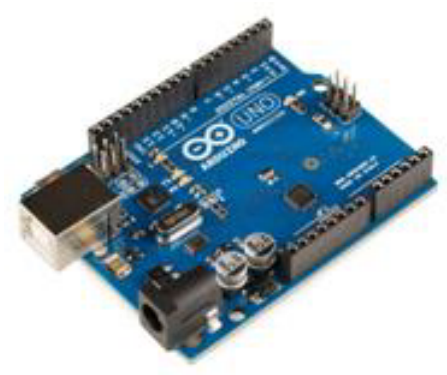

Figure 6. Arduino Uno

The wireless module used in this project is Bluetooth HC-06 module. HC-06 is a Bluetooth serial module that is used to convert the serial port into Bluetooth. This Bluetooth module is using Serial Port Protocol (SPP). It has $2.4 \mathrm{GHz}$ radio transceiver and baseband. This module also works on low power operation, 1.8 to $3.6 \mathrm{~V} \mathrm{I} / \mathrm{O}$. It also has a feature that it will auto-connect to the last device on power as default. HC-06 is used to connect with the Arduino Uno microcontroller. This allows the microcontroller board on the wheelchair to receive command signals wirelessly from the PC or smartphone. Figure 7 shows the HC-06 Bluetooth module.

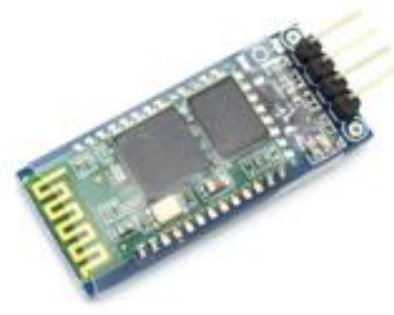

Figure 7. HC-06 Bluetooth Module

Emotiv EPOC [17] headset is the EEG device manufactured by the company of Emotiv. This headset is a multi-channel with high resolution portable EEG device for the purpose of brain-computer interface (BCI). This headset contains 14 channels which means 14 electrodes to capture the EEG signal from the brain. It has the most electrodes among the commercialized EEG device in the market. This device uses a single ADC with sequential sampling method. The sampling rate is at 128 samples per second. Emotiv EPOC headset is built in with digital 5th order Sinc filter and digital notch filters at $50 \mathrm{~Hz}$ and $60 \mathrm{~Hz}$. The battery of the headset is rechargeable LiPoly battery and the battery life can lasts for 12 hours. The wireless connectivity of the headset is using Bluetooth. The feature of Emotiv EPOC headset is it is able to translate the brainwaves which in EEG form into the metrics that can be easily understood. These metrics are translated by using different detection suite and they are known as facial expression, performance metrics and mental commands. The Facial Expression detection suite can detect the real-time facial expression of the user. Performance metrics is used to analyze the user's real time emotional states and monitor the user's state of mind. The Mental Command detection suite is used to read in and interpret the user's thought and intent. The mental commands are push, pull, 
left, right, and etc. The Mental Command detection suite is very useful for interpreting the user's mental command in order to control the electrical wheelchair movement. Figure 8 shows the Emotiv EPOC headset.

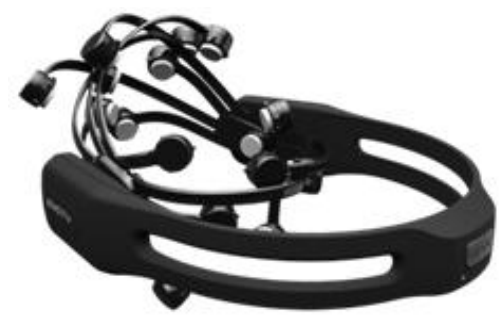

Figure 8. Emotiv EPOC Headset

\subsection{Hardware}

For the purpose of programming the Arduino Uno microcontroller, Arduino Integrated Development Environment (IDE) [18] is utilized to compile and upload the program code to the microcontroller. The advantages of this IDE are the provided software is open-source and only requires simple $\mathrm{C}$ language for the programming. Besides, it consists of various built-in functions which are very useful in most of the applications, for example analogWrite() which is the output PWM function.

The wheelchair is designed to be operated in two modes, which is the normal electrical mode that is triggered by switches and the brain controlled mode that is using the BCI method. The design of the program in flowchart is shown in Figure 9.

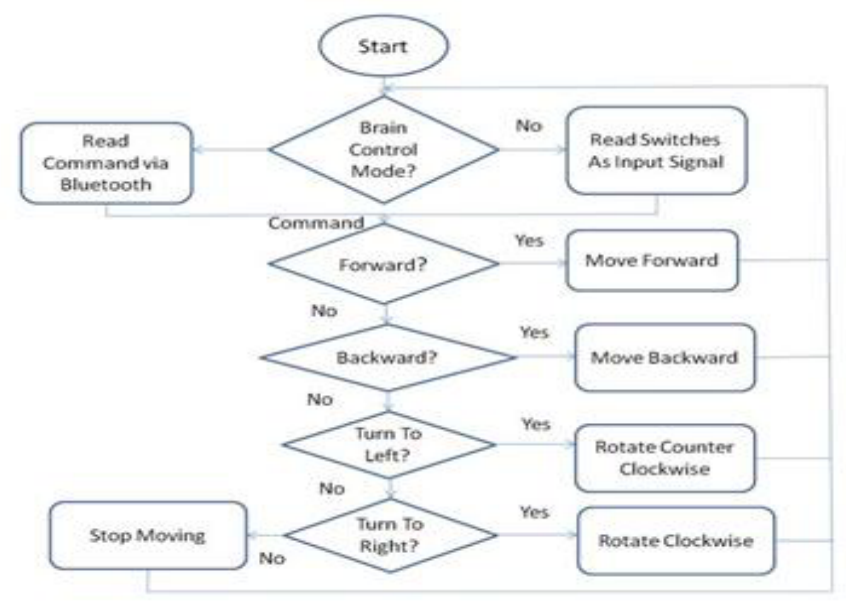

Figure 9. Flowchart of Microcontroller Program

Microsoft Visual C\# [19] is the IDE (Integrated Development Environment) that supports Visual C\# with a full-featured code editor and compiler. This IDE is used to write the Windows based application program for conducting several tasks. The first task is to receive the EEG data that transmitted out from Emotiv EPOC headset. Then, it processes the EEG signal. Lastly, it sends the electrical command signal out to the microcontroller by using Bluetooth wireless communication. The algorithm of the PC application in flowchart is shown in Figure 10. 


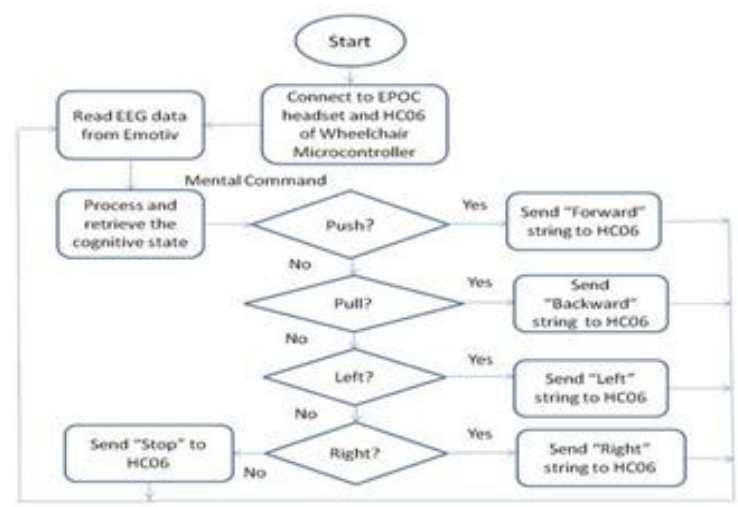

Figure 10. Flowchart of Microsoft Visual C\# Application Program

The program uses Emotiv API (Application Program Interface) to interface with the Emotiv EPOC headset. The EmoEngine in this API is used to communicate with the Emotiv EPOC headset, receives pre-processed EEG data, execute post-processing on the data and convert the detection results into another structure which is known as Emostate. Emostate is a data structure that consists of the state of the Emotiv detections, which are the user's facial, emotional and cognitive state. By analyzing the cognitive state, it is able to determine the mental command which is thinking by the user. Output signal is generated as string according to these mental commands (e.g. left, right and etc.). Then, the output signal is sent out to the wheelchair's microcontroller via Bluetooth.

\section{Results and Discussion}

As result, an electrical wheelchair which is able to function as a common electrical wheelchair is developed.

Windows-based Application for this project is written as shown in figure below. This program is able to read and process the EEG data from the Emotiv EPOC headset into mental command. After processing, this program sends out the command signal to the connected HC-06 on the microcontroller board of the wheelchair. In the end, the electrical wheelchair is able to move according to the user's desire.

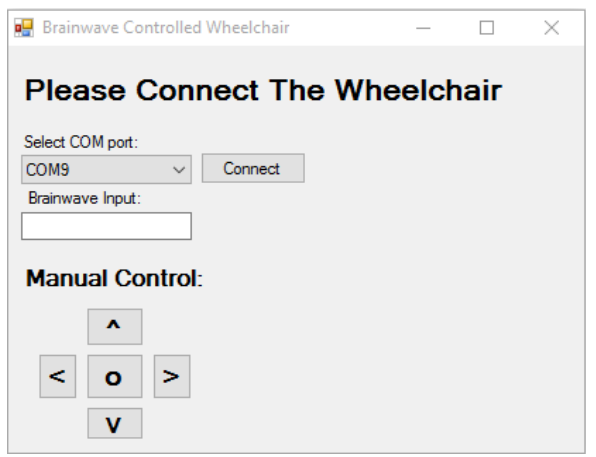

Figure 11. GUI for EEG Controlled Wheelchair

By using this application, the electrical wheelchair can be directly controlled by human brain. The results shows that the processed EEG data does not provide $100 \%$ accuracy according to the human mental command. However, it can achieve up to $90 \%$ of accuracy. The testing result for mental commands which conducted by 5 users are shown in table below: 
Table 1. Test Result for Mental Command

\begin{tabular}{|l|l|l|l|l|l|l|}
\hline Command/User & 1 & 2 & 3 & 4 & 5 & Total \\
\hline Forward & $5 / 10$ & $6 / 10$ & $8 / 10$ & $8 / 10$ & $4 / 10$ & $31 / 50$ \\
\hline Backward & $9 / 10$ & $8 / 10$ & $9 / 10$ & $9 / 10$ & $7 / 10$ & $42 / 50$ \\
\hline Left & $6 / 10$ & $7 / 10$ & $9 / 10$ & $8 / 10$ & $8 / 10$ & $38 / 50$ \\
\hline Right & $8 / 10$ & $9 / 10$ & $7 / 10$ & $9 / 10$ & $8 / 10$ & $41 / 50$ \\
\hline Total & $28 / 40$ & $30 / 40$ & $33 / 40$ & $34 / 40$ & $27 / 40$ & \\
\hline
\end{tabular}

Therefore, this shows that the BCI (brain-computer interface) for the electrical wheelchair is successfully established.

\section{Conclusion}

The main objective of this project is to construct an electrical wheelchair which can be directly controlled by the brain of the user. At the end of this project, an electrical wheelchair which can be operated in normal wheelchair mode and brainwave control mode is successfully developed. The brainwave controlled wheelchair can be controlled by most of the people. Besides, this project also contributes a new and effective solution for the physically disabled people or the patients who suffered from neuromuscular disorder to regain their mobility.

\section{References}

1. Public Service Department Malaysia, "What Is The Definition Of Senior Citizens In Malaysia?" [Online]. Available: http://www.jpapencen.gov.my/english/senior_citizen.html. [Accessed: 01Dec-2013].

2. Department of Social Welfare Malaysia, "Bhg. B 10 - Pendaftaran Orang Kurang Upaya,” 2009. [Online]. Available: http://www.jkm.gov.my/file/file/Statistic_2009/BHG B 10 PENDAFTARAN ORANG KURANG UPAYA.pdf. [Accessed: 20-Jan-2016].

3. Department of Social Welfare Malaysia, "Bhg. B 10 - Pendaftaran Orang Kurang Upaya," 2010. [Online]. Available: http://www.jkm.gov.my/file/file/Statistic_2010/BHG B 10 PENDAFTARAN ORANG KURANG UPAYA.pdf. [Accessed: 20-Jan-2016].

4. Department of Social Welfare Malaysia, "Bhg. B 10 - Pendaftaran Orang Kurang Upaya," 2011. [Online]. Available: http://www.jkm.gov.my/file/file/Statistic_2011/BHG B $10 \quad$ PENDAFTARAN ORANG KURANG UPAYA.pdf. [Accessed: 20-Jan-2016].

5. Department of Social Welfare Malaysia, "Bhg. B 10 - Pendaftaran Orang Kurang Upaya,” 2012. [Online]. Available: http://www.jkm.gov.my/file/file/Statistic_2012/BHG B 10 PENDAFTARAN ORANG KURANG UPAYA.pdf. [Accessed: 20-Jan-2016].

6. Department of Social Welfare Malaysia, "Bhg. B 10 - Pendaftaran Orang Kurang Upaya," 2013. [Online]. Available: http://www.jkm.gov.my/file/file/Statistic_2013/BHG $\quad$ B $10 \quad$ PENDAFTARAN ORANG KURANG UPAYA.pdf. [Accessed: 20-Jan-2016].

7. L. Bi, X. A. Fan, Y. Liu "EEG-Based Brain-controlled Mobile Robots: A survey", HumanMachine Systems, 43, 161-176, (2013).

8. A. Nijholt, D. Tan, G. Pfurtscheller, C. Brunner, J.del R. Mill'an, B. Allison, B. Graimann, F. Popescu, B. Blankertz, and K.-R. M"uller, "Brain-computer interfacing for intelligent systems.", IEEE Intelligence System, 23, 72-79. (2008).

9. WebMD. "Positron Emission Tomography (PET)."[Online]. Available: http://www.webmd.com/cancer/lymphoma/positron-emission-tomography. [Accessed: 9-Sep2014]. 
10. T. F. Collura. "EEG Frequency Bands." The Measurement, Interpretation, and Use of EEG Frequency Bands. (1997).

11. E. Niedermeyer \& B. F L. Silva. "Electroencephalography: Basic Principles, Clinical Applications and Related Fields." (2005).

12. K. H. Solanki, H. Pujara. "Brainwave Controlled Robot.", International Research Journal of Engineering and Technology (IRJET), 2, 609 -612. (2015).

13. K. Yendrapalli, S. S. N. P. K. Tammana The "Brain Signal Detection for Controlling the Robot.", International Journal of Scientific Engineering and Technology, 3, 1280-1283. (2014).

14. B. Rebsamen, C. Guan, H. Zhang, C. Wang, C. Teo, M. H. Ang, Jr., and E. Burdet, "A brain controlled wheelchair to navigate in familiar environments.", IEEE Trans. Neural Syst. Rehabil, 18, 590-598. (2010).

15. K. R. M"uller and B. Blankertz, "Toward noninvasive brain-computer interfaces,” IEEE Signal Processing Magazine, 23, 125-128. (2006).

16. A. Griffith, "An Exploration of the OpenEEG Project", C.H.G.Wright's BioData Systems, 2006.

17. Emotiv, " Brain Computer Interface \& Scientific Contextual EEG.", 2014. [Online]. Available: http://www.emotiv.com. [Accessed: 20-Jan-2016]

18. Arduino LLC, “Arduino Software IDE.” [Online]. Available: http://www.arduino.cc. [Accessed: 27-Aug-2015].

19. "Microsoft Visual C\#," Microsoft, 1989. [Online]. Available: http://msdn.microsoft.com [Accessed: 27-Aug-2015. 